\title{
OVERVIEW OF INFRARED SOLAR PHYSICS
}

\author{
JOHN T. JEFFERIES \\ National Solar Observatory, National Optical Astronomy Observatories, * \\ P. O. Box 26732, Tucson, Arizona 85726, U.S.A.
}

\begin{abstract}
A brief overview of the field of infrared solar physics is given with particular reference to the topics to be considered during the Symposium.
\end{abstract}

Key words: infrared: stars - Sun: atmosphere - Sun: general

\section{Introduction}

My task is to set a stage for what is to follow during the rest of the week, to convey some sense of solar infrared astronomy - what it is, what it has achieved, where it is going, and why it is important. I shall try to do that - but selectively and briefly, as is appropriate for an overview.

I think that this is the first major meeting on solar IR astronomy and we are, of course, gratified that the IAU thought it sufficiently important to grant it the status of a Symposium. Clearly I agree with their assessment; it is important. It is also an exciting area to be associated with, as it gives us a new perspective on nature, and fresh insights into old problems - often overturning our preconceptions and prejudices in the process. We will hear a lot about a wide range of science which IR studies have illuminated - of new light on models of the quiet Sun, new insights on atomic excitation, new horizons in solar magnetometry, new ways to look at solar oscillations, new potential for studying solar inhomogeneities, new perspectives on the outer atmosphere derived from eclipse observations, new levels of precision in abundance determinations. Some old friends also reappear in new garments - such as the 10830 line of helium which derives new vitality from improved observational techniques - though it seems that we still struggle, as we have for 40 years at least, to find a satisfying accounting for it. We shall hear plenty about all these things, and more, as the week unfolds.

So the solar IR is vigorous and wide-ranging in its importance. A number of specific characteristics combine to make it so, and I may perhaps take a minute or two to review these, dwelling first on the continuum and second on the lines.

\section{The Infrared Continuum}

The first thing to say about the IR continuum is that there is a lot of it. Our Symposium defines it as covering the range from $1 \mu \mathrm{m}$ to $1 \mathrm{~mm}$ or so. That is a factor of 1000 in wavelength, compared with the factor of less than 2 which we have to work with in the visible. The same physical processes control the continuum opacity over nearly all this range - namely free-free interactions involving an electron and a neutral $\mathrm{H}$ atom or a positive ion. We understand these processes, we know quite precisely their dependence on wavelength and particle density. The wide range of opacity which the IR opens up allows us to span a correspondingly wide range of

* Operated by the Association of Universities for Research in Astronomy, Inc., under cooperative agreement with the National Science Foundation. 


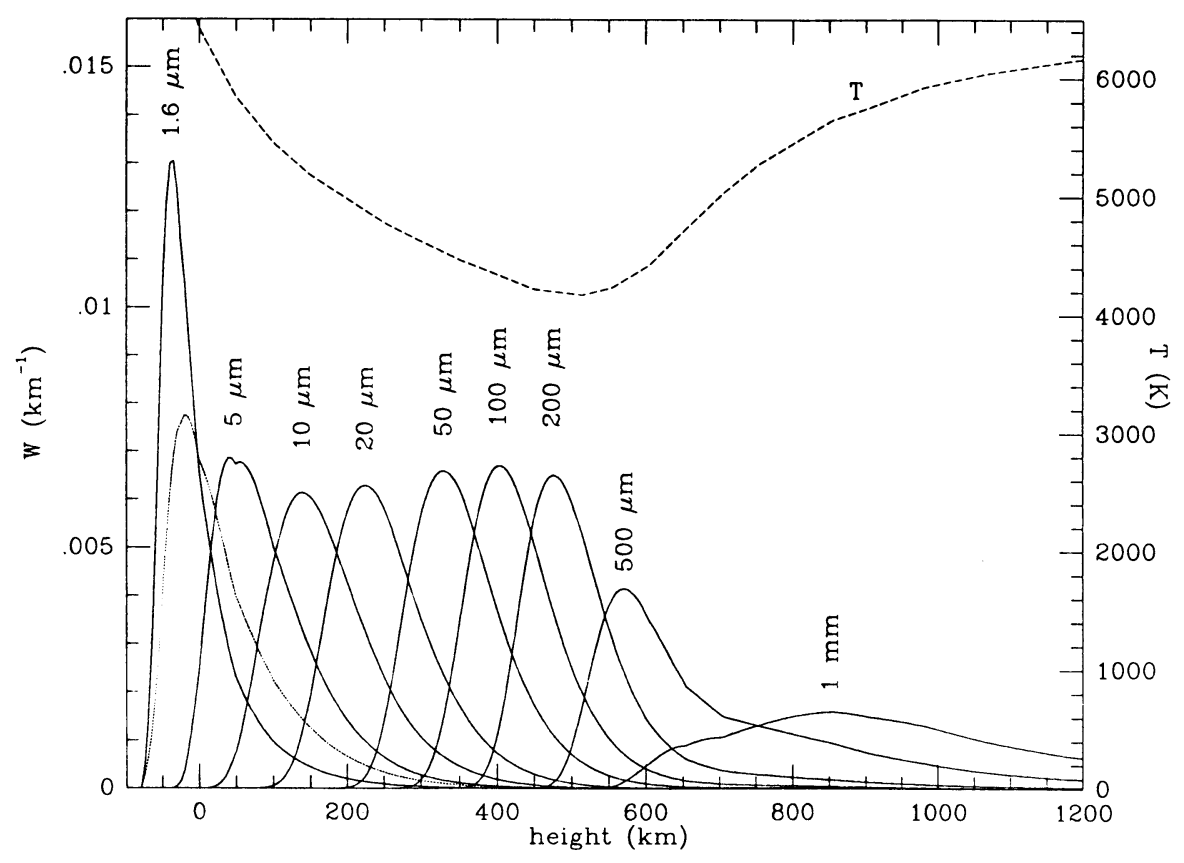

Fig. 1. Weighting functions for several wavelengths in the infrared for the VAL IIIC model atmosphere. The dotted curve is for $500 \mathrm{~nm}$ continuum radiation and the dashed curve shows the temperature distribution for the model.

atmospheric conditions as we scan through the IR. This is illustrated in Figure 1, which shows the contribution functions for a selection of wavelengths from 1.6 to $800 \mu \mathrm{m}$, for a standard solar model, VAL III-C, (Vernazza et al., 1981), whose temperature distribution is shown at the top of the Figure. Over this range we sample from the lowest accessible level in the photosphere up through the temperature minimum - more than $1000 \mathrm{~km}$ as compared to the $40 \mathrm{~km}$ or so which is accessible to us in the visible continuum. We can, of course, go further into millimeter wavelengths using the new facilities on Mauna Kea (the Caltech sub-mm Observatory and the JCMT) to which solar astronomers have been granted access - Ewell et al. (1992), Lindsey et al. (1990), Lindsey and Jefferies (1991), and Lindsey et al. (1992). Thus there are very good reasons, as Figure 1 shows, why modelling of the solar atmosphere has drawn so heavily on observations at longer wavelengths, and why one would continue to place emphasis on improving them in accuracy, in spatial resolution, and in the wavelength range which is covered. However, to achieve this diagnostic power has not been straightforward. Much of the range is only accessible from above the upper atmosphere or from space, as Figure 2 illustrates, so that balloons, aircraft, or space vehicles are needed to cover the full wavelength range of the infrared. To achieve the level of spatial resolution needed for critical studies of the Sun, we shall have to go to much larger apertures (or baselines) than are 


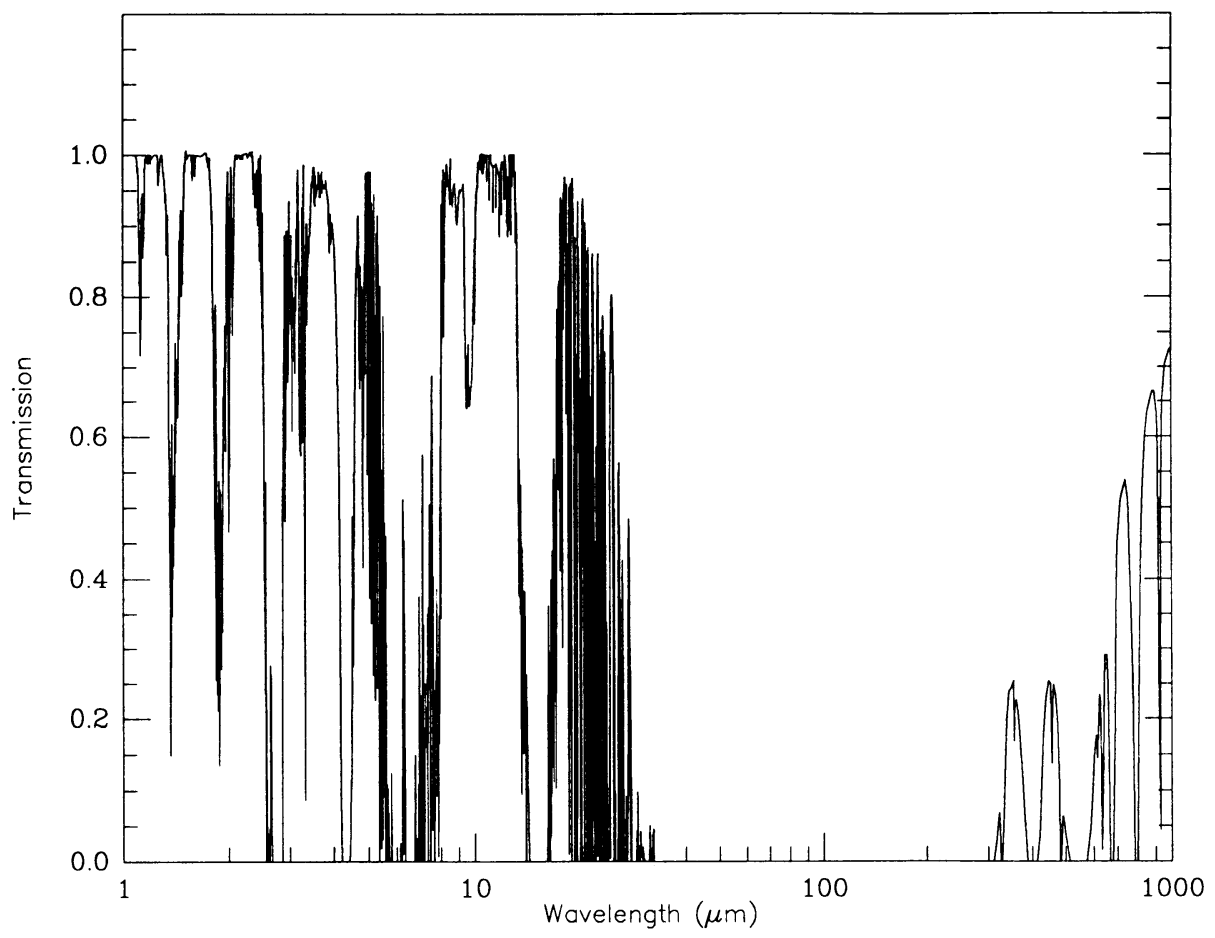

Fig. 2. Atmospheric transmission for Mauna Kea (1.2 mm water-vapor) - from Traub and Stier (1976).

presently available. Even so, much has been achieved in experiments such as those of Eddy et al. (1969), Rast et al. (1978), at the shorter wavelengths, and by such observers as Noyes, Beckers and Low (1968), Clark et al. (1971), Kundu (1971), Gezari et al. (1973), Lindsey and Hudson (1976), in the sub-millimeter to millimeter wavelength region. A first idea of the potential which increased resolution will hold for clarifying the structure of the Sun over the wide range of heights spanned by the 'infrared' is given in the images shown in Figure 3 taken from the JCMT at $350 \mu \mathrm{m}$ and $1.3 \mathrm{~mm}$ - Lindsey and Jefferies (1991). Clearly the continuum intensity over the solar disk from the short IR through to the millimeter wavelengths is a fundamental and powerful diagnostic tool for inferring conditions in the Sun, and we shall hear a lot about this during the Symposium.

Another incisive application of IR continuum observations of the Sun is found in eclipse studies - both of the extreme limb and of the outer reaches of the atmosphere which can be especially well observed on those occasions. These aspects, too, will be well represented in our discussions here. As an illustration, Figure 4, compiled from Lindsey et al. (1986), Roellig et al. (1991), and Lindsey et al. (1992), shows how the Sun's intensity varies at the limb for several wavelengths in the IR, together with the predictions of a standard model. Clearly there is a discrepancy between 


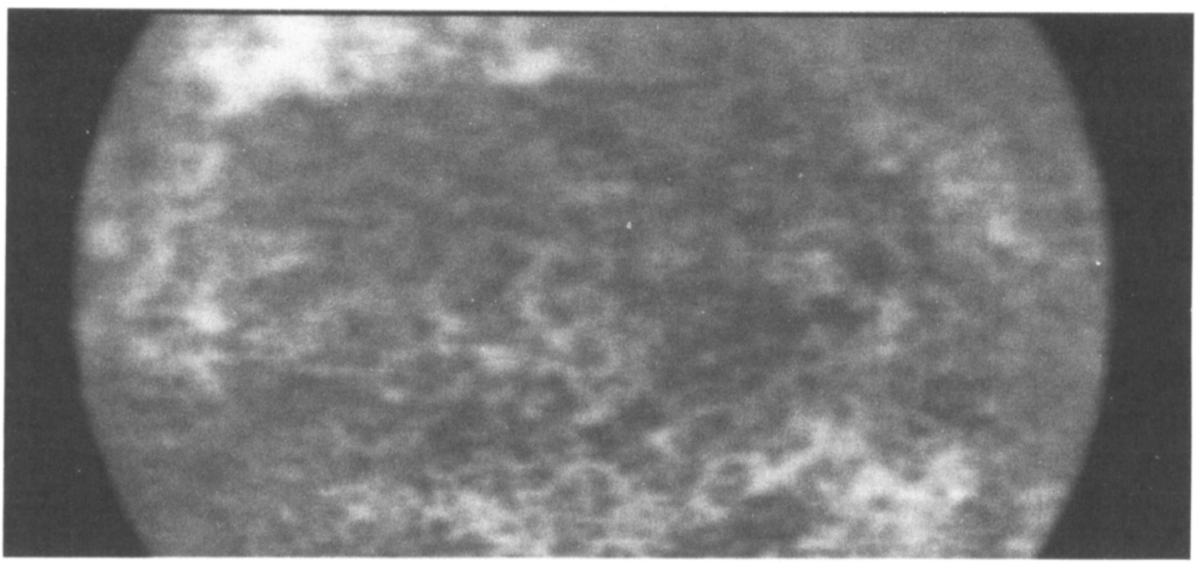

b

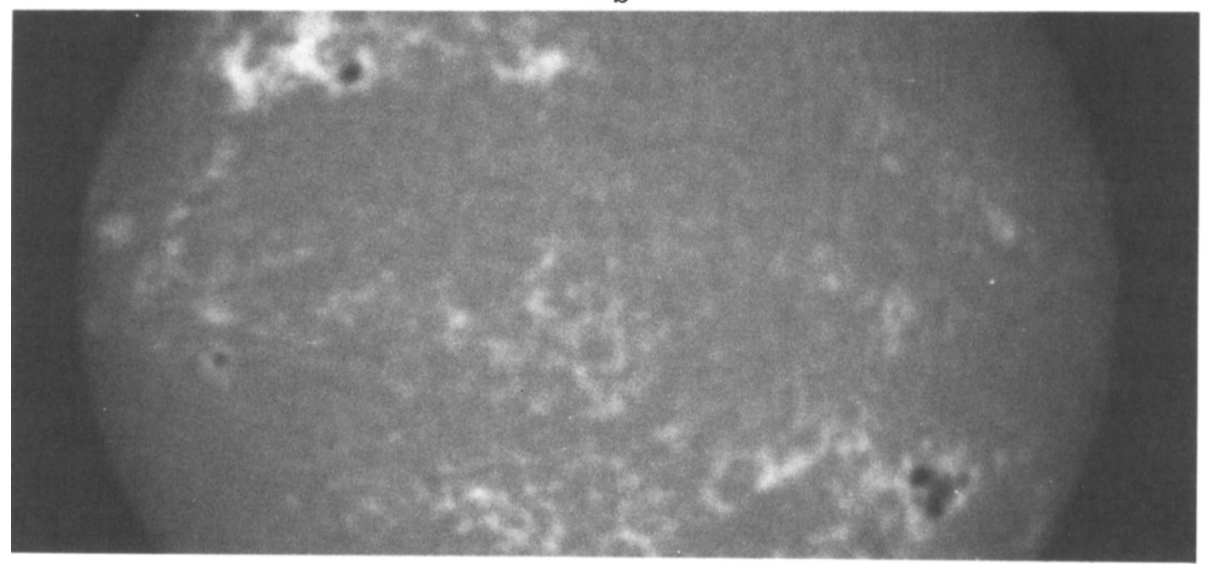

Fig. 3. Solar images at $850 \mu \mathrm{m}($ panel $a)$, and in the Ca II K-line (panel $b$ ).

the observational data and the theory which, in fact, only grows worse as the wavelength increases up through $1.3 \mathrm{~mm}$. This is probably a result of our neglect of inhomogeneities, as millimeter observers have been pointing out since the 1950's see, for example, Hagen (1954), Coates (1958) Simon and Zirin (1969), Lantos and Kundu (1972), Kalaghan (1974).

There is a more subtle, and as yet barely touched, diagnostic possibility which the IR offers, namely its potential for inferring the nature of the inhomogeneities found in the solar atmosphere. Because, as I mentioned earlier, the IR flux from the Sun reflects the temperature linearly, the radiation from a composite region will contain, coded in its intensity, information about the temperature of the different components of the source - in the nature of a linear average over the fluctuations. This is a peculiar advantage of the IR - by contrast, at shorter wavelengths, the hotter elements tend to dominate the measured intensity giving us little to work 


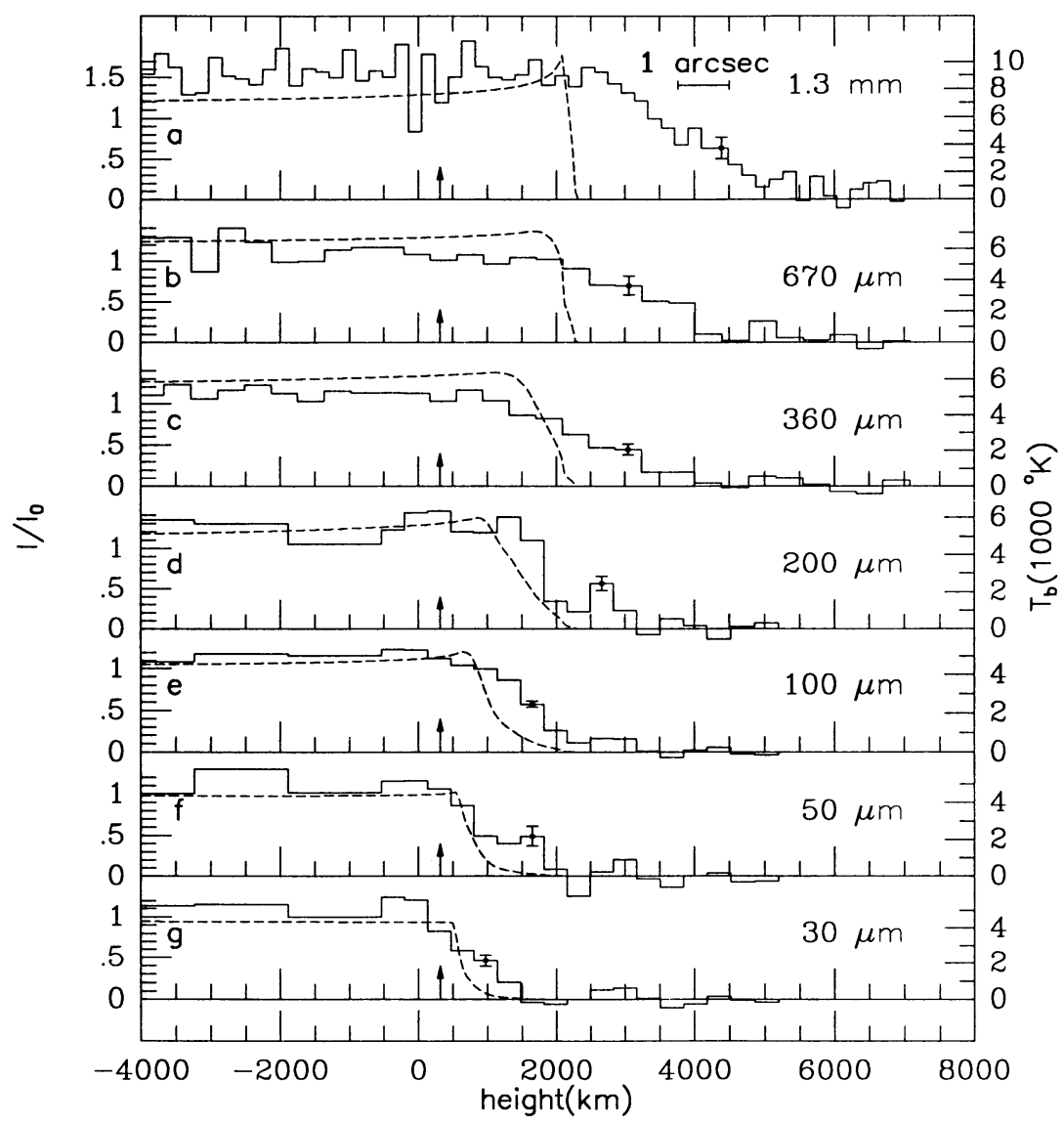

Fig. 4. Limb brightness profiles determined by occultation at total solar eclipses. Panel $a$ is for 1991 July 11 (Lindsey et al. 1992,); $b$ and $c$ are for 1987 March 18 (Roellig et al. 1991); and $d, e, f$, and $g$ were for the 1981 July 31 eclipse (Lindsey et al. 1986.)

with in determining the temperature characteristics of the composite structure. There are encouraging prospects, indeed, that with infrared data at several wavelengths we will be able to infer some essential characteristics of this sub-telescopic structure.

So the IR continuum is a flexible and powerful diagnostic tool. Its formation is simple and well understood, so we can make predictions with a good deal of confidence. We have an extended wavelength range to work with, so we can span a wide range of conditions. And perhaps we shall be able to make progress on inhomogeneous diagnostics. 


\section{The Infrared Line Spectrum}

What about the lines? What can they tell us? One clear and decided advantage to the IR is in vector magnetometry where the higher splitting opens a range of new possibilities. An early application of the IR advantage was made by Harvey and Hall (1975), using the $1.56 \mu \mathrm{m}$, line of $\mathrm{Fe} \mathrm{I}$; this approach was subsequently used by Stenflo et al. (1987) and has been developed, at the hands of Rabin and his collaborators (see, e.g., Rabin and Graves, 1989), into a very powerful instrument at the McMath telescope. The discovery of the $12-\mu \mathrm{m}$ emission lines by Brault and Noyes (1984) opened a further avenue for high-accuracy magnetometry of the Sun which has been developed by Deming and his collaborators (Deming et al. 1988), and by Hewagama et al. (1989), also using the McMath telescope. In particular the large splitting often allows us to measure the magnetic field directly in situations where only the magnetic flux is measured using visible line magnetometers. That this difference is critical is due to the fact that most (if not all) of the solar flux is found to be concentrated in flux-tubes and the area they occupy in different solar features is at best poorly known - thus we cannot with any confidence estimate the actual field strength without going into the IR. In fact we may be able to go further and obtain the distribution of field strengths in the sampled area, especially using lines in the $12-\mu \mathrm{m}$ range where the splitting is very large. This whole area of research is very active and full of promise; we will hear much more about this in the future.

To turn to another aspect - high resolution IR spectroscopy from the ground gave us the $12-\mu \mathrm{m}$ lines - Brault and Noyes (1984) - with their strong emission cores and a host of intriguing problems to go along with them. In Figure 5, which is actually taken from the ATMOS spectra (Farmer and Norton, 1989), we show some other lines in the same sequence. Many of us had thought that all these lines, arising in transitions between high-lying levels of neutral $\mathrm{Mg}$, would be formed in LTE. Adjacent levels must be closely coupled, by electron collisions, with each other and with the ionized state and so we would seem to have all the conditions needed for an LTE population. Also the core emission seemed just to be reflecting the temperature increase into the chromosphere. It was really a pretty and consistent picture and early computations - Lemke and Holweger (1987) - seemed to bear this out. But this conclusion was wrong; as studies by Chang et al. (1991), and Carlsson et al. (1992) have shown, these lines are not formed in LTE in the Sun and the core emission has nothing to do with the chromospheric temperature increase. However, I cannot say that I understand the theoretical results as deeply as I would like and I look forward to hearing, at this meeting, the latest on this intriguing question. In the meantime we have the data on several other lines of $\mathrm{Mg}$ from the beautiful $A T M O S$ spectra- and it will be a further challenge to the theorists to explain all of it at the same time. It was, of course, the emission character of the $12-\mu$ mlines that excited interest in their formation. No such interest would have attached to them had they been in absorption. Yet the super-excitation, which underlies the emission phenomenon, is driven by the local conditions where the line is formed, and for a differnt line, formed at a different location, we shall as likely find an under-excitation, giving rise to an absorption line-equally far from LTE, equally 

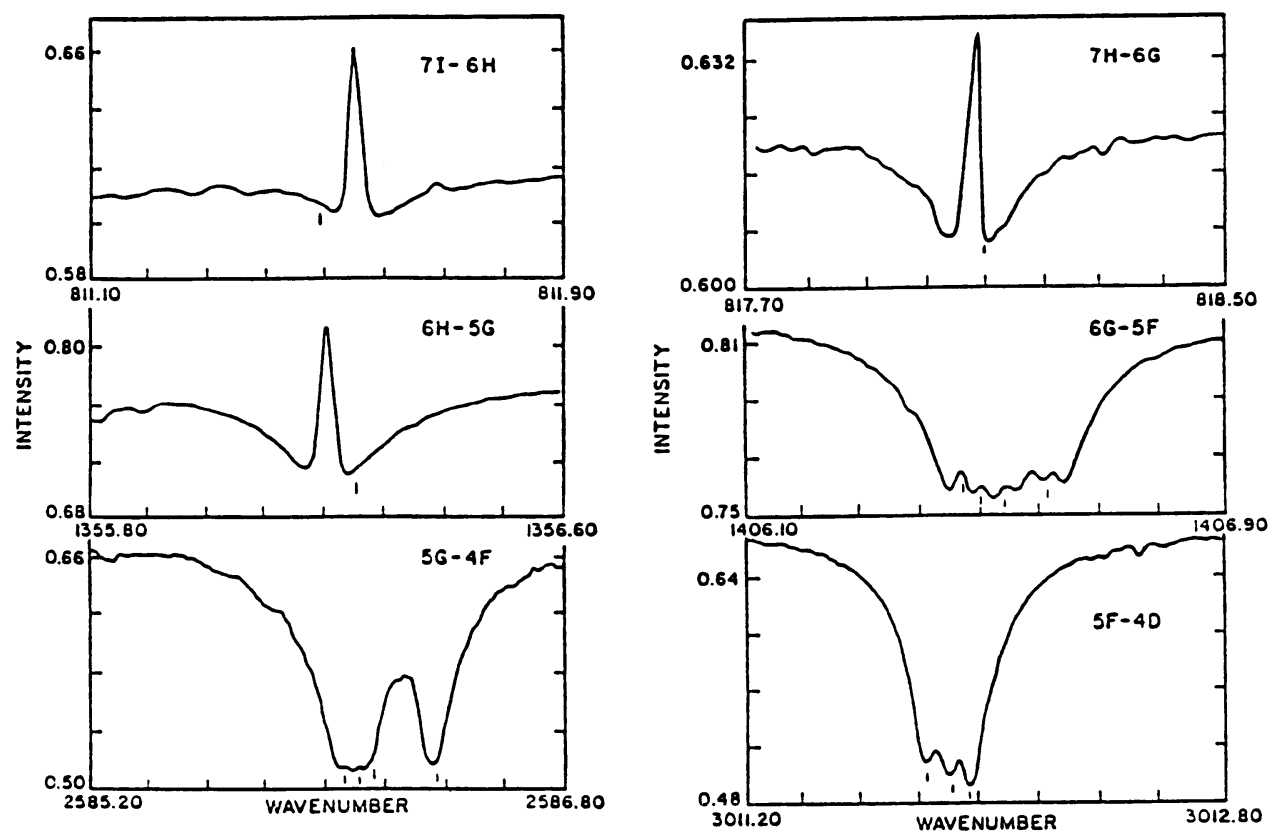

Fig. 5. ATMOS profiles of several lines of $\mathrm{Mg} \mathrm{I}$.

anomalous, and leading equally to error if interpreted as in LTE. The Mg I studies have shown us that, when dealing with atomic or molecular lines in the mid- to far-IR, whether in emission or absorption, we must approach their interpretation with great care, and not trust inferences (e.g. of temperature or abundance) based on LTE without a most careful analysis to ensure the validity of this assumption.

\section{Concluding Remarks}

There are many other aspects of the solar IR spectrum which we shall be hearing about during the week - determination of isotopic abundances, solar oscillations, high-lying lines of $\mathrm{H}$, the 10830 line - all exciting new work opened up by the IR.

One final note. The solar IR is a burgeoning field with a bright and promising future - and as such it brings its own demands for the facilities, instruments, and support needed to develop the potential of the field. Some ideas will come out at this meeting - e.g., the plans for a bigger McMath, new arrays, adaptive optics to combat the diffraction disadvantage of the IR. There can be little doubt that the pressure for such specialized capabilities will only increase, and the arguments for satisfying them become more compelling, as the successes of solar IR astronomy mount. I look forward to a stimulating few days learning more about those successes. 


\section{References}

Brault, J.W. and Noyes, R.W.: 1984, Astrophys. J. (Letters) 269, 61.

Carlsson, M., Rutten, R.J., and Shchukina, N.G.: 1992, Astron. Astrophys. 253, 567.

Chang, E.S., Avrett, E.H., Mauas, P.J., Noyes, R.W., and Loeser, R.: 1991 Astrophys. J. (Letters) $379,79$.

Clark, T.A., Courts, G.R., and Jennings, R.E.: 1971, Phil. Trans. A. 270, 55.

Coates, R.J.: 1958, Astrophys. J. 128,83.

Deming, D., Boyle, R.J., Jennings, D.E., and Wiedemann, G.: 1988, Astrophys. J. 333, 978.

Eddy, J.A., Lena, P.J., and MacQueen, R.M.: 1969, Solar Phys. 10, 330.

Ewell, M.W., Zirin, H., Jensen, J., and Bastian, T.: 1993, these proceedings.

Farmer, C.B., and Norton, R. (eds.): 1989, A High-Resolution Atlas of the Infrared Spectrum of the Sun and Earth from Space, vol. 1, The Sun, NASA RP-1224.

Gezari, D.Y., Joyce, R.R., and Simon, M.: 1973, Astron. Astrophys. 26, 409.

Hagen, J.P.: 1954, J. Geophys. Res. 59, 158.

Harvey, J.W., and Hall, D.N.B.: 1975, Bull. Amer. Astron. Soc. 7, 459.

Hermans L., and Lindsey, C.: 1986, Astrophys. J. 310, 448.

Hewagama, T., Jennings, D. Deming, D., Boyle, R., and Zipoy, D.M.: 1989, Bull. Amer. Astron. Soc. 21, 839.

Kalaghan, P.M.: 1974, Solar Phys. 39, 315.

Kundu M.K.: 1971, Solar Phys. 21, 130.

Lantos, P., and Kundu, M. 1972, Astron. Astrophys. 21, 119.

Lemke, M., and Holweger, H.: 1987, Astron. Astrophys. 173375.

Lindsey, C.A., and Hudson, H.: 1976, Astrophys. J. 203, 753.

Lindsey, C.A., Becklin, E.E., Orrall, F.Q., Werner, M.W., Jefferies, J.T., and Gatley, I.I.: 1986, Astrophys. J. 308, 448.

Lindsey, C.A., Jefferies, J.T., Clark, T.A., Harrison, R.A., Carter, M.K., Watt, G., Becklin, E.E., Roellig, T.L., Braun, D.C., Naylor, D.A., and Tomkins, G.J.: 1992, Nature 392, 739.

Lindsey C.A., and Jefferies, J.T.: 1991, Astrophys. J. 383, 443.

Noyes, R. W., Beckers, J. M. and Low, F. J.: 1968, Solar Phys. 3, 36.

Rabin, D.M. and Graves J.E.: 1989, Bull. Amer. Astron. Soc. 21, 854.

Rast, J., Kneubühl, F.K., and Müller, E.A.: 1978 Astron. Astrophys. 68, 229.

Simon, M., and Zirin, H.: 1969, Solar Phys. 9, 317.

Stenflo, J.O., Solanki, S.M., and Harvey, J.W.: 1987 Astron. Astrophys. 173, 167.

Traub W., and Stier M.T.: 1987, Appl. Optics 15, 364.

Vernazza, J.E., Avrett, E.H., and Loeser, R.: 1981 Astrophys. J. Suppl. 45 , 635. 